\title{
A NUMERICAL ANALYSIS OF BULK-BARRIER DIODES
}

\author{
E. LANGER and S. SELBERHERR \\ Institut für Physikalische Elektronik, TU Wien, Gußhausstraße 27, A-1040 Wien, Austria
}

and

H. MADER

Siemens AG, Zentrale Forschung und Entwicklung, Otto-Hahn-Ring 6, D-8000 München 83, West Germany

(Received 1 June 1981; in revised form 9 September 1981)

\begin{abstract}
A selfconsistent numerical analysis of bulk-barrier diodes (BBD) is presented. The principal way of operation of a BBD is explained. A computer program which can accurately model second order effects is used to achieve basic understanding of the internal electric behaviour of a BBD. The distributions of the relevant physical quantities in the interior of a BBD are discussed. Measured and simulated characteristics which show good agreement, owing to the carefully modeled physical parameters, are compared. The sensitivity of electrical properties to minute variations of the doping profile is presented. This can only be done by a program simulating the characteristics of a BBD. The advantages and disadvantages of a BBD compared to a standard silicon diode and a Schottky diode are discussed.
\end{abstract}

\section{INTRODUCTION}

Bulk-barrier diodes (BBD) are relatively new devices which promise to have impact on integrated circuits henceforth. Current flow in BBD's is essentially accomplished by majority carriers and controlled by a "bulk-barrier" which is adjustable with standard technological steps. At first glance the BBD is comparable to a Schottky diode which is also a majority carrier device. As a remarkable difference the barrier of a Schottky diode is located at the metal-semiconductor interface and not in the bulk, and is not controllable by technological steps.

This paper deals with a numerical analysis of the BBD, which has been performed by a computer program. In Section 2 the structure and the principle operation of this new type of diode are explained. The physical model the computer program is based on is outlined in Section 3. The underlying equations are discussed and all assumptions are justified. The formulae which are used to model the physical parameters are explained in Section 4. It is not the objective of this paper to describe the numerical solution of the equations. We should like to mention this fact explicitly, because our analysis is just performed one-dimensionally - this type of analysis for diodes has already been published in $1968[1,2]$-and recently published papers already deal with two-dimensional transient analysis [3-6] or even three-dimensional analysis [7]. However, our one-dimensional semiconductor simulation program is just as good for some practical applications, with the additional advantage of accurately modeled physical parameters[8]. In Section 5 the internal distributions of the physical variables at various bias conditions are discussed to get comprehensive insight of the effects which are responsible for the correct operation of the BBD. A comparison of simulation and measurement is presented in Section 6 which verifies the applicability of the physical model. Furthermore the sensitivity of the electric properties of the BBD on uncertainties of the doping profile and consequently of the technological process is demonstrated. Numerical device analysis yields excellent results for this kind of investigation whereas the correct interpretation of experimental measurements is rather cumbersome.

\section{THE PRINCIPLE OPERATION OF BULK-BARRIER DIODES}

To explain the fundamental principle of operation we take a BBD with a $p^{+} n p$ doping profile (Fig. 1). The $n$-layer is relatively thin, so that without an applied voltage the whole $n$-layer is depleted of free electronsthe $p^{\prime} n p$-diode is "punched through" [9]. If one applies a positive voltage between the $p$ - and $p^{+}$-layer, the $p^{+} n$ junction-henceforth called the first junction-is reverse biased and the $n p$-junction-the second junction-is forward biased. As the doping in the $p^{+}$-layer is higher than in the $n$-layer, the depletion region of this reverse biased diode extends mainly into the $n$-layer. As a result of punch through a hole current flows from the $p$ - to the $p^{+}$-layer at relatively low voltages; the BBD is forward biased. The knee voltage of the BBD can be controlled by the doping level and the thickness of the $n$-layer alone; i.e. by technological steps.

If one applies a negative voltage between the $p$ - and $p^{+}$-layer, the second junction is reverse biased. This junction is, owing to the low substrate doping, able to block, because the depletion region extends mainly into the substrate; the BBD is reverse biased.

Analytic investigations of the above mentioned effects can be found in references $[10,11]$ and a physical analy. sis of an analogous structure is given in Refs. $[12,13]$. 


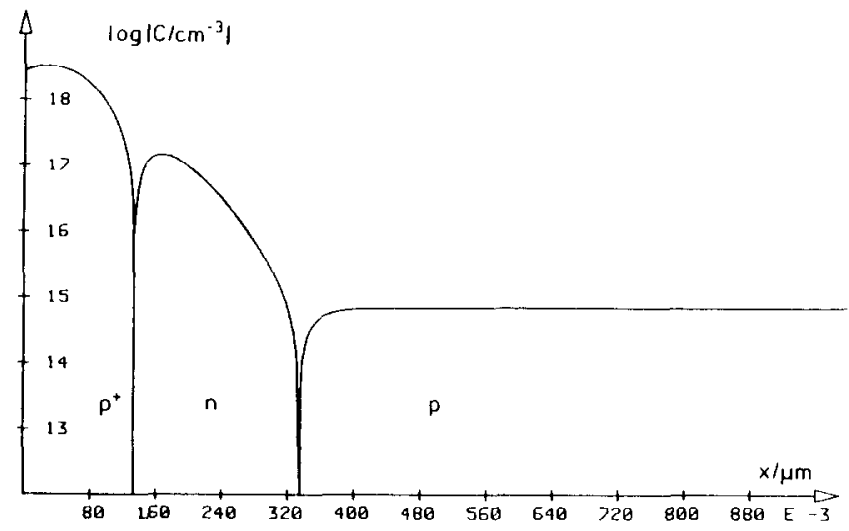

Fig. 1. Doping profile of the investigated $p^{+} n p$ BBD.

\section{THE PHYSICAL MODEL}

The physical model is based on the well-known fundamental semiconductor equations [14] which have to be solved in order to analyze accurately carrier transport in an arbitrary semiconductor structure. These eqns (1)-6) represent mathematically a nonlinear two-point boundary value problem which can be solved numerically with standard methods.

Poisson's eqn

$$
\frac{\mathrm{d}}{\mathrm{d} x}\left(\epsilon \cdot \frac{\mathrm{d} \psi}{\mathrm{d} x}\right)=-q \cdot\left(p-n+N_{D}{ }^{+}-N_{A}{ }^{-}\right)
$$

continuity eqns

$$
\begin{array}{ll}
\text { for electrons } & \frac{\mathrm{d} J_{n}}{\mathrm{~d} x}=q \cdot R \\
\text { for holes } & \frac{\mathrm{d} J_{p}}{\mathrm{~d} x}=-q \cdot R
\end{array}
$$

\section{current relations}

$$
\begin{aligned}
\text { electrons } J_{n} & =-q \cdot\left(\mu_{n} \cdot n \cdot \frac{\mathrm{d} \psi}{\mathrm{d} x}-D_{n} \cdot \frac{\mathrm{d} n}{\mathrm{~d} x}\right) \\
\text { holes } J_{p} & =-q \cdot\left(\mu_{p} \cdot p \cdot \frac{\mathrm{d} \psi}{\mathrm{d} x}+D_{p} \cdot \frac{\mathrm{d} p}{\mathrm{~d} x}\right)
\end{aligned}
$$

heat flow eqn

$$
k \cdot \frac{\mathrm{d} T}{\mathrm{~d} x}+Q=0
$$

In the following a few assumptions which are based on physical considerations will be discussed. The reason for making assumptions is not only to ease the solution of the equations, but to speed up the computer program with negligible loss of accuracy. It is, therefore, of paramount importance to verify carefully the validity range of the model.

\section{Boltzmann statistics}

Boltzmann statistics (7) and (8) is sufficient to describe carrier density distributions. With this assumption it is impossible to simulate devices with extremely heavy doping. However, up to doping levels of $5 \times 10^{19} \mathrm{~cm}^{3}$ the error introduced by Boltzmann statistics is commonly acceptable. It is to be noted that bandgap-narrowing can certainly be modeled by using a doping dependent intrinsic number. If it is desired to analyze transport phenomena with extremely heavy doping, one has to use Fermi statistics, and thus, the basic eqns (1)-(6) have to be modified slightly $[15,16]$.

$$
\begin{array}{ll}
\text { electrons } & n=n_{i} \cdot \exp \left(\frac{\psi-\psi_{n}}{U_{T}}\right) \\
\text { holes } & p-n_{i} \cdot \exp \left(\frac{\psi_{p}-\psi}{U_{T}}\right) .
\end{array}
$$

Einstein's relations

The Einstein-Nernst relations (9) and (10) hold for describing the diffusion constants. This assumption is directly coupled with Boltzmann statistics and the original form of the basic eqns (4) and (5), since for their derivation no difference has been made between electron temperature and lattice temperature [17]

$$
\begin{array}{ll}
\text { electrons } & D_{n}=\mu_{n} \cdot U_{T} \\
\text { holes } & D_{p}=\mu_{p} \cdot U_{T} .
\end{array}
$$

\section{Permittivity}

The permittivity is isotropic and constant (11). This assumption is not all a restriction in case of silicon which, as an elemental semiconductor, has a diamond lattice[17].

$$
\epsilon_{\mathrm{si}}=\text { constant }
$$

\section{Total ionization}

The impurities are treated as being totally ionized (equation 12). Within a desired temperature range of $250 \mathrm{~K}$ to $450 \mathrm{~K}$ it is not necessary to include partial ionization[17], thus no extra effort has to be made to determine the inhomogeneity of Poisson's equation and neutral impurity scattering does not have to be included in mobility modeling.

$$
N_{D}{ }^{+}=N_{D} ; \quad N_{A}{ }^{-}=N_{A} ; \quad N_{D}-N_{A}=C .
$$


All contacts can be treated as classical ohmic contacts; the space charge is therefore vanishing and carrier densities are in thermal equilibrium.

\section{THE PHYSICAL PARAMETERS}

In order to obtain accurate simulation results, one has to model carefully a set of physical parameters. The most important parameters which will be discussed in detail are the carrier mobilities which depend on quite a few physical effects and the carrier recombination/generation phenomena.

\section{Thermal voltage}

The thermal voltage is by definition linearly temperature dependent and simply given by eqn (13).

$$
U_{T}=\frac{k_{B} \cdot T}{q}
$$

\section{Intrinsic number}

The intrinsic number is modeled as being temperature dependent [18] and doping dependent [19] with formulae (14) and (15). Thus moderate degeneracy can be simulated as the effect of bandgap-narrowing is included with the doping dependent intrinsic number. Not too much sophistication is used to model the temperature dependence of the effective masses in eqn (14) as these effects arc as yct not too well explored.

$$
\begin{aligned}
n_{i}(T)= & 6.43 \cdot 10^{15} \cdot\left(\frac{T}{K}\right)^{3 / 2} \\
\cdot & \left.\exp \left(\frac{\frac{4.07 \cdot(T / K)^{2}}{T / K+1108}-6730}{T / K}\right)\right)\left[\mathrm{cm}^{-3}\right] \\
n_{i}(N, T)= & n_{i}(T) \cdot \exp \left(\frac { 5 2 . 2 K } { T } \cdot \left(\ln \frac{N}{N_{0}}+\right.\right. \\
+ & \left.\left.\left(\left(\ln \frac{N}{N_{0}}\right)^{2}+0.5\right)^{1 / 2}\right)\right)\left[\mathrm{cm}^{-3}\right]
\end{aligned}
$$

with

$$
N=N_{D}^{+}+N_{A}^{-} ; \quad N_{0}=10^{17} \mathrm{~cm}^{-3}
$$

\section{Lattice scattering}

For an adequate mobility expression one has to take into account several scattering mechanisms. The trivial one is temperature dependent lattice scattering eqn (16) which is well analyzed [20]. A simple power law [21] can be used to describe the temperature dependence. The indices $n$ and $p$ of the constants denote the values for electrons and holes, respectively

$$
\begin{array}{cl}
\mu_{0}(T)=A \cdot(T / K)^{-8}\left[\mathrm{~cm}^{2} / \mathrm{Vs}\right] \\
A_{n}=7.12 \cdot 10^{8} \mathrm{~cm}^{2} / \mathrm{Vs} & A_{p}=1.32 \cdot 10^{8} \mathrm{~cm}^{2} / \mathrm{Vs} \\
g_{n}=2.3 & g_{p}=2.2
\end{array}
$$

\section{Ionized impurity scattering}

The lattice mobility is reduced through the scattering process at ionized impurities. There are a number of papers dealing with impurity scattering and one principally has to distinguish between theoretically based models [20,22] and heuristic formulae [23, 24] which are verified for quantitative correctness. The heuristic formulae are commonly not as complicated as the theoretical ones, when the same accuracy is considered, and are, therefore, to be preferred. Equation (17) is a formula which models temperature dependent ionized impurity scattering and electron-hole scattering[25]. Electron-hole scattering is extremely important in low doped regions where high injection takes place

$$
\begin{aligned}
& \mu_{N}(N, T)=a \cdot \mu_{0}(T)+b \cdot(1-a)\left[\mathrm{cm}^{2} / \mathrm{Vs}\right] \\
& a=\frac{1}{1+(T / 300 K)^{c} \cdot\left(N / N_{0}\right)^{d}} \\
& N=0.67 \cdot\left(N_{D}^{+}+N_{A}^{-}\right)+0.33 \cdot(n+p) \\
& b_{n}=55.24 \mathrm{~cm}^{2} / \mathrm{Vs} \quad b_{p}=49.7 \mathrm{~cm}^{2} / \mathrm{Vs} \\
& c_{n}=-3.8 \quad c_{p}=-3.7 \\
& d_{n}=0.73 \quad d_{p}=0.7 \\
& N_{0 n}=1.072 \cdot 10^{17} \mathrm{~cm}^{-3} \quad N_{0 p}=1.606 \cdot 10^{17} \mathrm{~cm}^{-3} \text {. }
\end{aligned}
$$

\section{Velocity saturation}

In regions with a high electric field component parallel to current flow, the drift velocity saturation phenomenon has to be taken into account. This can be done by further reducing the carrier mobilities. Equation (18) is a heuristic formula describing this physical effect with a slightly temperature dependent saturation velocity and Mathiessens weight $[21,26]$

$$
\mu_{N E}(E, N, T)=\frac{\mu_{N}(N, T)}{\left(1+\left(\mu_{N}(N, T) \cdot|E| / v_{s}\right)^{h}\right)^{1 / h}}\left[\mathrm{~cm}^{2} / \mathrm{Vs}\right]
$$

$$
\begin{aligned}
v_{s n} & =1.53 \cdot 10^{9} \cdot\left(\frac{T}{K}\right)^{-0.87} \mathrm{~cm} / \mathrm{s} \\
h_{n} & =2.57 \cdot 10^{-2} \cdot(T / K)^{0.66} \\
v_{s p} & =1.62 \cdot 10^{8} \cdot\left(\frac{T}{K}\right)^{-0.52} \mathrm{~cm} / \mathrm{s} \\
h_{p} & =0.46 \cdot(T / K)^{0.17}
\end{aligned}
$$

\section{Recombination}

To simulate satisfactorily transfer phenomena of majority-and minority-currents in just a simple diode, or avalanche breakdown at high electric field and current limitation at high injection, it is an absolute necessity to model carrier recombination and generation as carefully as possible (eqn 19)

$$
R=R_{\text {rec }}-R_{\text {gen }}=R_{\mathrm{SRH}}+R_{I}+R_{\text {Aug }}
$$

\section{Thermal recombination}

Thermal generation/recombination can be modeled by a so-called Shockley-Read-Hall term (eqn 20). The carrier lifetimes are to be simulated as being doping 
dependent [27]

$$
\begin{aligned}
& R_{\mathrm{SRH}}=\frac{p \cdot n-n_{i}^{2}}{\tau_{p} \cdot\left(n+n_{i}\right)+\tau_{n} \cdot\left(p+n_{i}\right)}\left[\mathrm{cm}^{-3} \mathrm{~s}^{-1}\right] \\
\tau= & \tau_{0} /\left(1+N / N_{0}\right) \\
N= & N_{D}{ }^{+}+N_{A} \\
\tau_{0 n}= & 3.95 \cdot 10^{-5} \mathrm{~s} \quad \tau_{0 p}=3.52 \cdot 10^{-5} \mathrm{~s} \\
N_{0 n}= & 7.1 \cdot 10^{15} \mathrm{~cm}^{-3} \quad N_{0 p}=7.1 \cdot 10^{15} \mathrm{~cm}^{3} .
\end{aligned}
$$

\section{Avalanche generation}

Impact ionization can be modeled by an exponentially field dependent generation term. The constants in eqn (21) are basically taken from [28]. It should be noted that this form of simulating avalanche is relatively crude compared to more exact considerations but the underlying physical principles are so complex that a trade-off in accuracy and complexity has to be reached.

$$
R_{I}=-\frac{1}{q} \cdot\left(\alpha_{n}(E) \cdot\left|J_{n}\right|+\alpha_{p}(E) \cdot\left|J_{p}\right|\right)\left[\mathrm{cm}^{-3} \mathrm{~s}^{-1}\right]
$$

$$
\begin{array}{rlrl}
\alpha(E) & =A \cdot \exp (-b /|E|) \\
A_{n} & =7.03 \cdot 10^{5} \mathrm{~cm}^{-1} \quad A_{p}=1.582 \cdot 10^{6} \mathrm{~cm}^{-1} \\
b_{n} & =1.23 \cdot 10^{6} \mathrm{~V} / \mathrm{cm} & b_{p}-2.036 \cdot 10^{6} \mathrm{~V} / \mathrm{cm}
\end{array}
$$

\section{Auger recombination}

To analyze high injection conditions, Auger recombination has to be included as an antagonism to avalanche generation. Already the use of simple formulae like (22) [29] gives in general satisfactory results. However, it should be noted that major numerical difficulties can arise when treating the Auger recombination term inconsistently.

$$
\begin{aligned}
& R_{\text {Aug }}=\left(C_{n} \cdot n+C_{p} \cdot p\right) \cdot\left(p \cdot n-n_{i}^{2}\right)\left[\mathrm{cm}^{-3} \mathrm{~s}^{1}\right] \\
& C_{n}=2.8 \cdot 10^{-31} \mathrm{~cm}^{6} / \mathrm{s} \quad C_{p}=9.9 \cdot 10^{-32} \mathrm{~cm}^{6} / \mathrm{s}
\end{aligned}
$$

\section{Thermal conductivity}

Thermal conductivity is modeled by (23). The temperature dependence is relatively weak [30] and does not introduce nonlinearity problems in (6)

$$
k(T)=3110 \cdot(T / K)^{-4 / 3}[\mathrm{~W} / \mathrm{Kcm}]
$$

\section{Heat generation per unit volume}

There are essentially two possibilities to model the heat generation per unit volume $Q^{\prime}$ which is the derivative of the inhomogeneity in (6). Relation (24) has been proposed in Ref. [31] and is extremely simple to use. However, if the orientation of the electric field and the current density is not the same, this formula leads to an unrealistic local heat sink. In Ref. [32] a more sophisticated derivation of the thermal current density is given which avoids the mentioned problems, but unfortunately the corresponding formula (25) is more complicated to evaluate numerically

$$
\begin{aligned}
& Q^{\prime}=E \cdot\left(J_{n}+J_{p}\right)=-\frac{\mathrm{d} \psi}{\mathrm{d} x} \cdot\left(J_{n}+J_{p}\right)\left[\mathrm{W} / \mathrm{cm}^{3}\right] \\
& Q^{\prime}=\frac{\mathrm{d}}{\mathrm{d} x}\left(\psi_{n} \cdot J_{n}+\psi_{p} \cdot J_{p}\right)\left[\mathrm{W} / \mathrm{cm}^{3}\right]
\end{aligned}
$$

\section{RESULTS OF THE NUMERICAL ANALYSIS}

For our investigations we used a BBD with a $p^{+} n p$ doping profile, a length of $470 \mu \mathrm{m}$ and an area of $0.5 \mathrm{~mm}^{2}$. As the doping profile determines the behaviour of the BBD in an extremely critical way, the modeling of the doping profile has been performed carefully by using SUPREM-II-the Stanford University Process Engineering Models program[33]. As input for this process simulation program technological process data of an experimental diode were used so that a consistently modeled doping profile could be guaranteed. The results of SUPREM-II were directly used as input for our device simulation program.

Figure 1 shows the calculated doping profile of our investigated $\mathrm{BBD}$; one can read off a thickness of the implanted $n$-layer of about $200 \mathrm{~nm}$.

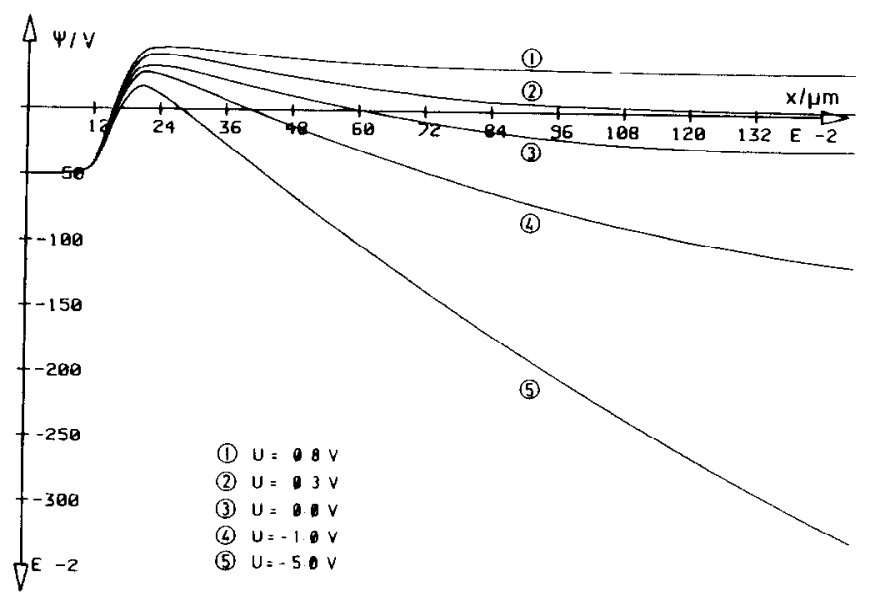

Fig. 2. Potential distributions. 
Figure 2 shows the potential distribution in the interior of the BBD at various operating points. One can easily extract from this figure that the barrier is vanishing with increasing bias. It is to be noted at this point that the blocking barrier at reverse bias conditions tends to become smaller too with increasing reverse bias which will certainly lead to an increase in current. The quantitative amount of the barrier lowering, however, does not affect the blocking capability at moderate reverse bias conditions.

Figure 3 shows the carrier density distributions at zero bias. The hole density is negligibly small in the $n$-layer but it can rise rather quickly to its equilibrium value. The electron density does not reach its equilibrium value in the $n$-layer, the BBD is, therefore, punched through already at zero bias.

Figures 4 and 5 show the carrier density distribution for a forward bias of 0.3 and $0.8 \mathrm{~V}$, respectively. The increase of the hole density is fairly pronounced. At a bias of $0.8 \mathrm{~V}$ in the whole device the hole density is larger than the electron density which is, owing to the punch through effect, vanishing in the $n$-layer; this is a typical behavior of a majority carrier device because the transfer current is nearly exclusively determined by the holes. The kink of the electron density at the first junction is due to thermal generation. This kink does not exist at $0.3 \mathrm{~V}$ since the magnitude of the hole density is not as large at this operating point.

Figures 6 and 7 show the carrier density distributions for a reverse bias of -1.0 and $-5.0 \mathrm{~V}$, respectively. One can see the depletion region of the second junction; it even extends out of locus bounds in Fig. 7. However, it is to be noted that the magnitude of the hole density, although it is small, increases with higher reverse bias which leads to the already mentioned increase in saturation current.

Figure 8 shows the mobility distribution for electrons and holes plotted for two different operating points; $0.8 \mathrm{~V}$ forward bias and $-5.0 \mathrm{~V}$ reverse bias. The most interesting fact is the local mobility maximum at reverse bias. At this locus the mobility reduction owing to impurity scattering is already less prominent and the mobility reduction owing to velocity saturation is still not active because the electric field is not yet large enough. At the reverse biased operating point one can see a local mobility minimum which is effected on the one hand by

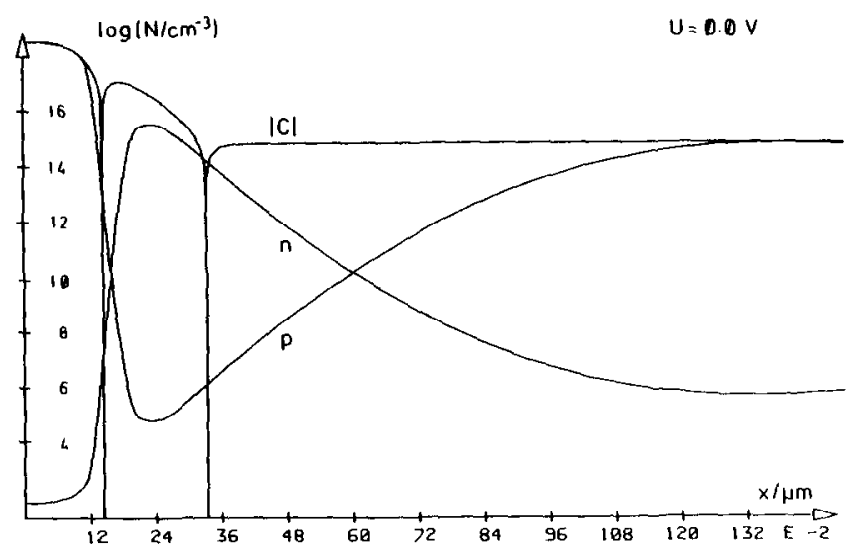

Fig. 3. Carrier distributions at zern hias.

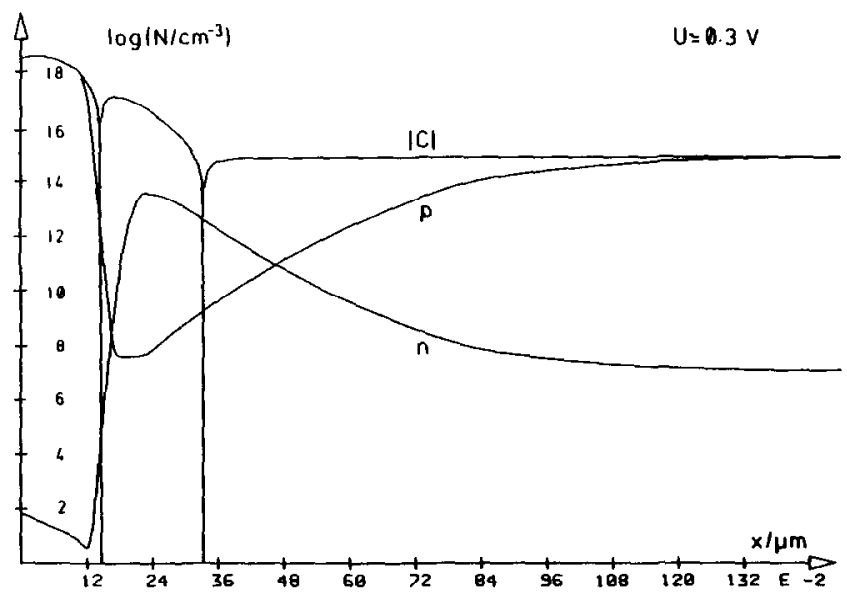

Fig. 4. Carrier distributions at $0.3 \mathrm{~V}$. 
322

E. LANGER et al.

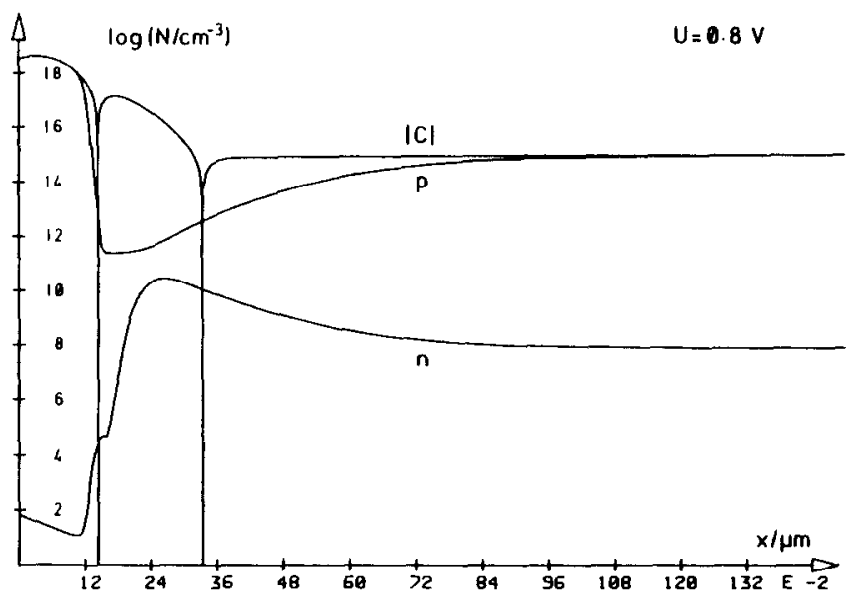

Fig. 5. Carrier distributions at $0.8 \mathrm{~V}$.

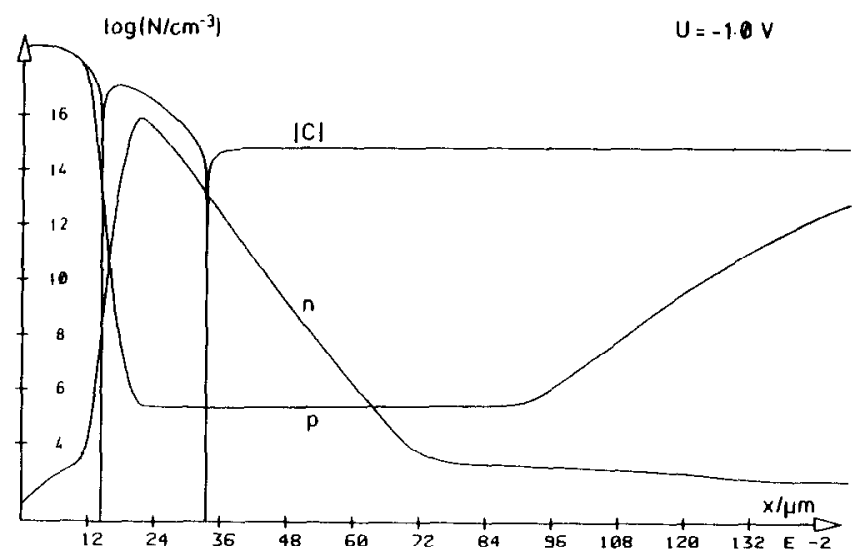

Fig. 6. Carrier distributions at $-1.0 \mathrm{~V}$.

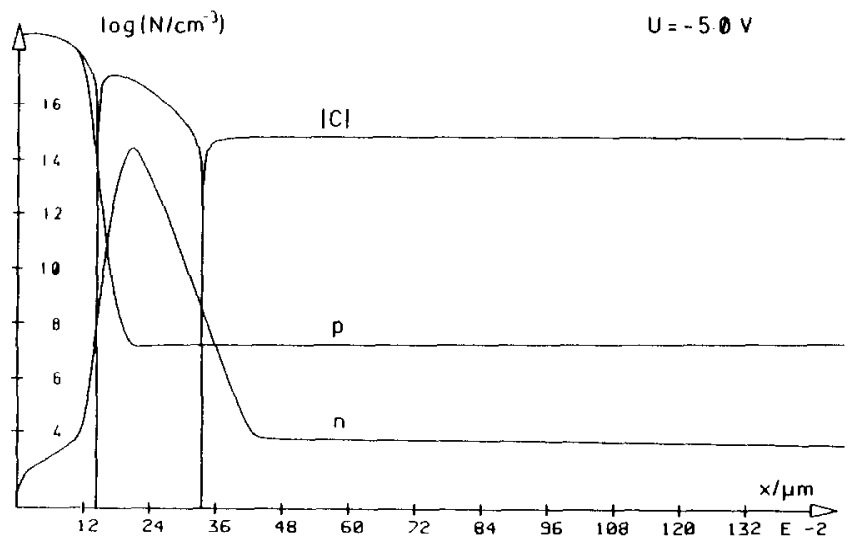

Fig. 7. Carrier distributions at $-5,0 \mathrm{~V}$. 


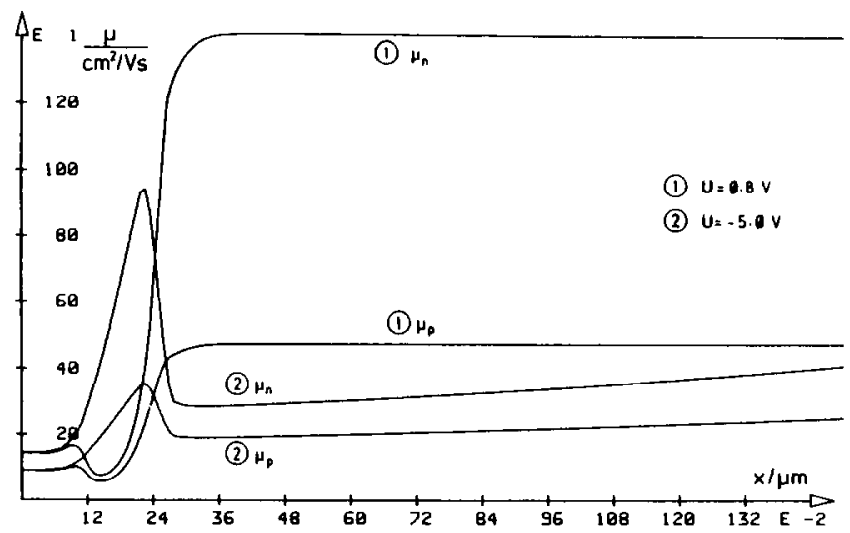

Fig. 8. Mobility distributions at 0.8 and $-5.0 \mathrm{~V}$.

velocity saturation owing to the high electric field and on the other hand by impurity scattering in consequence of the high doping concentration of the $p^{+}$-layer.

\section{COMPARISON WITH MEASUREMENT}

Figure 9 shows a comparison of the simulated and the measured characteristics of the BBD. The predicted diode behaviour is fully confirmed. The knee voltage is distinctly lower than for a standard silicon diode. The offset one can see for forward bias between simulation and measurement is too small to speak of bad agreement. This offset is due to the uncertainty of the doping profile as confirmed by Figs. 10 and 11.

Figure 10 shows the forward characteristics of the BBD drawn in a logarithmic scale to cover a larger bias range. Excellent agreement of simulation and measurement has been obtained. Also plotted in this figure are simulated characteristics with slightly changed doping parameters. The data of the characteristics are to be understood as changes of the implantation dose, i.e. $-5 \% \mathrm{P}$ means a reduction of the implantation dose of

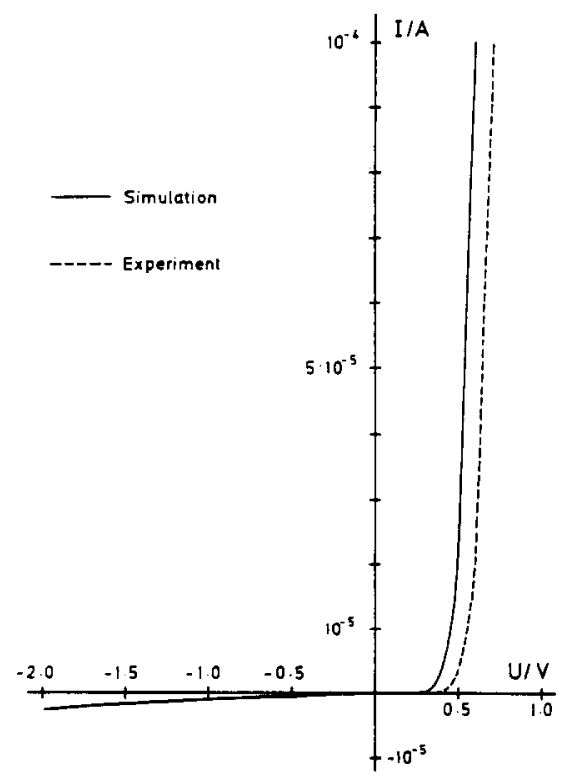

Fig. 9. Comparison of measured and simulated characteristics.

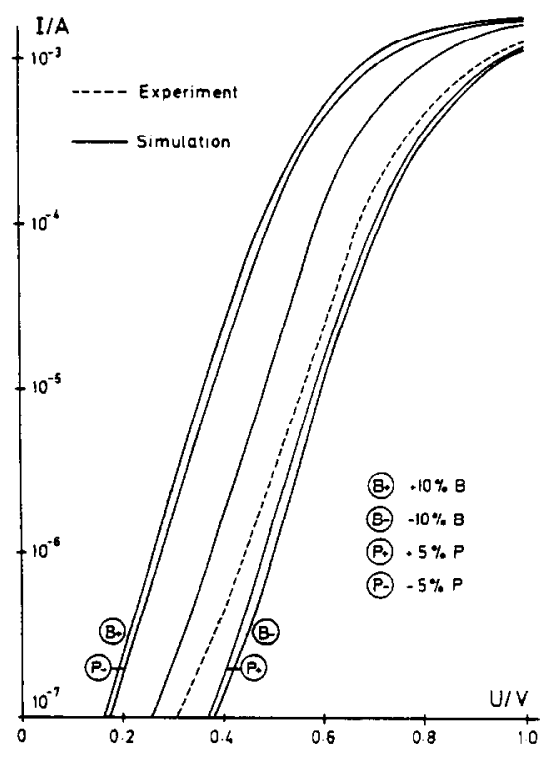

Fig. 10. Forward bias characteristics and sensitivity.

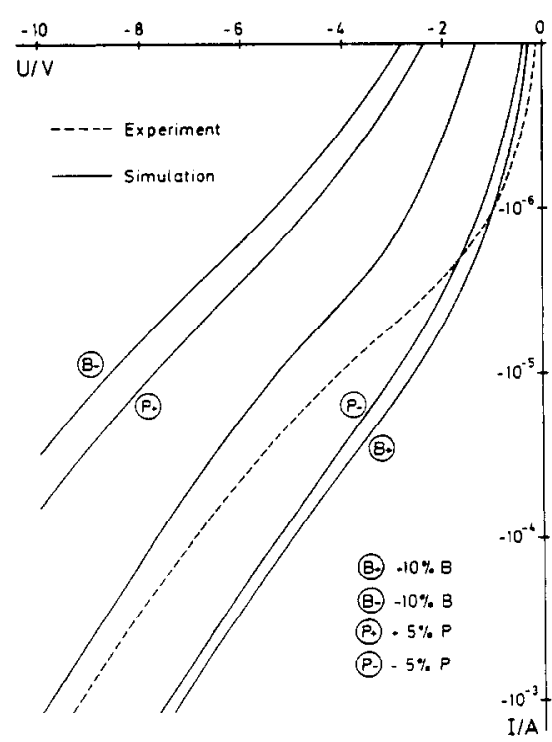

Fig. 11. Reverse hias characteristics and sensitivity. 
phosphorus by $5 \%$ compared to the value of the investigated $\mathrm{BBD}$. The shift of the characteristics owing to even these small changes is quite pronounced, so that there is no reason to regard this offset as a simulation error. As concluding remark it seems necessary to state that enormous care has to be taken in designing the fabrication process of a BBD to obtain the desired knee voltage. In spite of this critical sensitivity production has proved to be possible.

Figure 11 shows the blocking characteristics of the BBD in similar presentation as Fig. 10. One has an approximately exponential dependence of the saturation current on the bias. Small technological changes result in similar offsets as for forward bias. This offset is not visible in Fig. 9 owing to the linear scale. The blocking performance of a BBD is not as excellent as the one of a standard silicon diode. This fact has to be taken into account when designing a circuit.

\section{CONCLUISION}

When summarizing all advantages and disadvantages, there is certainly a wide field of applications for a BBD. BBD's are majority carrier devices with diode like characteristics. The knee voltage of BBD's can be controlled by standard technological steps which is a tremendous advantage compared to silicon diodes as well as to Schottky diodes which have in all other respects very similar properties. The switching speed of BBD's can be expected to be very high since the relevant time constant, the dielectric relaxation time, is in the order of picoseconds. Additionally the capacity characteristics and the differential resistance could be tuned with technological steps for special purpose applications.

Our computer program for simulating any type of silicon diodes is able to predict and analyse satisfactorily the behaviour of the BBD. This application is in fact much more complicated than the analysis of a standard silicon diode, because the BBD reacts extremely sensitively to a number of parameters. It is nevertheless possible to obtain good quantitative agreement of simulation results and measurements, because much emphasis has been laid on the adequate modeling of second order effects. The analysis of the thermal behaviour can be performed with this program too, although it has not been demonstrated in this paper, because it is a third order effect for the BBD. The computer program is available from the authors for just the handling fees.

Acknowledgements-This work has been supported by the "Fonds zur Förderung der wissenschaftlichen Forschung" (Projekt Nr. S22/11). The authors wish to thank Dipl. Ing. D. Schornböck and the whole staff of the computer centre for the excellent computer access, Prof. Dr. H. Pötzl for many useful discussions and Dipl.Ing. J. Machek for critically reading our manuscript.

\section{REFERENCES}

1. A. De Mari, Solid-St. Electron. 11, 33-58 (1968).

2. A. De Mari, Solid-St. Electron. 11, 1021-2053 (1968).

3. J. J. Barnes and R. J. Lomax, Electron. Lett. 11, 519-521 (1975).

4. E. M. Buturla and P. E. Cotrell, Solid-St. Electron. 23, 331-334 (1980).

5. P. E. Cotrell and E. M. Buturla, Proc. NASECODE I Conf., pp. 31-64 (1979).

6. O. Manck, Numerische Analyse des Schaltverhaltens eines zweidimensionalen bipolaren Transistors. Dissertation, Technische Hochschule Aachen, 1975.

7. E. M. Buturla, P. E. Cotrell, B. M. Grossman, K. A. Salsburg, M. B. Lawlor and C. T. McMullen, Proc. Int. Solid-St. Circuits Conf., pp. 76-77 (1980).

8. E. Langer, Numerische Simulation der Halbleiterdiode. Diplomarbeit, Technische Universität Wien (1980).

9. S. M. Sze, Physics of Semiconductor Devices. Wiley, New York (1969).

10. H. Mader, Kennlinien von Halbleiter-Vielschichtstrukturen. Dissertation, Technische Universität München (1976).

11. H. Mader, European Pat. No. 0003101979.

12. J. M. Shannon, Appl. Phys. Lett. 35, 63 (1979).

13. J. M. Shannon, Br. Pat. No. 157, 3309 (1977).

14. W. V. Van Roosbroeck, Bell Syst. Techn. J. 29, 560-607 (1950).

15. A. H. Marshak and R. Shrivastava, Solid-St. Electron. 22. 567-571 (1979).

16. K. M. Van Vliet, Solid-St. Electron. 23, 49-53 (1980).

17. K. Seeger, Semiconductor Physics. Springer, Wien (1973).

18. F. H. Gaensslen and R. C. Jaeger, Solid-St. Electron. 22, 423-430 (1979).

19. J. W. Slotboom and H. C. De Graaff, Solid-St. Electron. 19, 857-862 (1976).

20. S. S. Li and W. R. Thurber, Solid-St. Electron. 20, 609-616 (1977).

21. C. Jacoboni, C. Canali, G. Ottaviani and A. A. Quaranta, Solid-St. Electron. 20, 77-89 (1977).

22. P. P. Debye and E. M. Conwell, Phys. Rev. 93, 693-706 (1954).

23. D. A. Antoniadis, A. G. Gonzales and R. W. Dutton, J. Electrochem. Soc. 125(5), 813-819 (1978).

24. D. M. Caughey and R. E. Thomas, Proc. IEEE 52, 2192-2193 (1967).

25. W. L. Engl, O. Manck and A. W. Wieder, Process and Device Modeling for Integrated Circuit Design (Edited by F. Van de Wiele, W. L. Engl and P. G. Jepers), pp. 377-418. Noordhoff, Leyden (1977).

26. R. Jaggi and H. Weibel, Helv. Phys. Acta. 42, 631-632 (1969).

27. D. C. D'Avanzo, Modeling and Characterization of Shortchannel Double Diffused MOS Transistors. Stanford Technical Report No. G-201-6 (1980).

28. V. A. Temple and M. S. Adler, IEEE Trans. Electron Devices ED-22, 910 (1975).

29. J. Dziewior and W. Schmid, Appl. Phys. Lett. 31, 346 (1977).

30. P. D. Maycock, Solid-St. Electron. 10, 161-163 (1967).

31. S. P. Gaur and D. H. Navon, IEEE Trans. Electron Devices ED-23, $50-57$ (1976).

32. A. Chryssafis and W. Love, Solid-St. Electron. 22, 249-256 (1979).

33. D. A. Antoniadis, S. Hansen and R. W. Dutton, Suprem II-A Program for IC Process Modeling and Simulation. Stanford Technical Report No. 5019-2 (1978). 